American Journal of Applied Sciences 6 (9): 1758-1762, 2009

ISSN 1546-9239

(C) 2009 Science Publications

\title{
Optimal Power Multicast Problem in Wireless Mesh Networks by Using a Hybrid Particle Swarm Optimization
}

\author{
${ }^{1}$ Allam Maalla, ${ }^{1}$ Chen Wei and ${ }^{2}$ Haitham J. Taha \\ ${ }^{1}$ School of Information Engineering, \\ Wuhan University of Technology, Wuhan 430070, Hubei, China \\ ${ }^{1}$ School of Electrical and Electronic Engineering, \\ University Sians Malaysia, Pahang, Malaysia
}

\begin{abstract}
Problem statement: Most mobile nodes in a wireless ad hoc network are powered by energy limited batteries; the limited battery lifetime imposes a constraint on the network performance. Therefore, power consumption of network operations is critical and subject to optimization. Approach: A novel hybrid PSO was proposed for solving the minimum power multicast problem in wireless ad-hoc networks. The MPM problem has been mathematically formulated to a constrained optimization problem using hybrid PSO. Results: In results, we considered that the centralized algorithm requiring a global knowledge of the networks is impractical in large wireless networks, the distributed version of the centralized algorithm requiring multihop neighborhood information under the assumption that limited frequency band is available. Conclusion: A variety of simulations were conducted to examine the performance of the proposed approach and the results show that our algorithm consistently outperforms existing techniques.
\end{abstract}

Key words: Wireless networks, multicast, power consumption, hybrid PSO

\section{INTRODUCTION}

Wireless Mesh Networks (WMN) are multi-hop networks, composed of wireless links ${ }^{[1]}$, that are typically composed of static wireless mesh routers and potentially mobile mesh clients. The major advantage of multicast routing lies in its capability of saving network resources since only one copy of message needs to be transmitted over a link shared by paths leading to different destinations. One of the major concerns in ad hoc wireless networks is reducing node energy consumption. Several algorithms for constructing minimum power broadcast trees have been presented in ${ }^{[1-5]}$. Initial research on multicast routing mainly focused on the problem of finding a minimum Steiner tree. The Broadcast Incremental Power (BIP) algorithm proposed in $^{[2]}$ exploits wireless multicast advantage and provides better performance than the Minimum Spanning Tree (MST) and Shortest-Path Tree (SPT). Wieselthier et al. ${ }^{[3]}$ proposed the BIP algorithm, which is based on the greedy heuristic method and provides a very fast sub-optimal solution.

The usage of multiple radios significantly improves the capacity of the network by employing concurrent transmissions in the network ${ }^{[4,5]} \cdot \operatorname{In}^{[4]}$, low complexity, distributed and power efficient broadcasting schemes are proposed in the dense networks, which is approximated as a continuum of nodes. $\mathrm{In}^{[6]}$, a greedy filling algorithm is proposed for the minimum cost broadcast tree, where at each iteration the reliable node that maximizes the full rate of the unreliable nodes is found firstly and then the node reached from the found node with minimum transmission power is included in the broadcast tree. As demonstrated by ${ }^{[7]}$, the power consumption is dominated by communications. For the purpose of energy conservation, each node can (possibly dynamically) adjust its transmitting power, based on the distance to the receiving node and the background noise. An evolutionary approach using stochastic search is proposed by Marks et al. ${ }^{[8]}$, which suggests that improved results can be achieved over BIP algorithm at a higher computational cost. A swarm based Ant Colony System (ACS) algorithm is presented by Das et al. ${ }^{[9]}$, which is shown to be able to find better solutions in very little computation time.

In this study, a hybrid PSO was proposed for minimize the total energy consumption for the multicast session problem. The powerful search capability of $\mathrm{HPSO}^{[10]}$ is a key factor in improving the system performance in terms of the total consumption

Corresponding Author: Allam Maalla, School of Information Engineering, Wuhan University of Technology, Wuhan 430070, Hubei, China 
power. Simulation results demonstrate that the proposed approach outperforms the MPM algorithm and is comparable to the best reported results in the literature so far.

Related work: A significant body of research in MANETs has researched efficient network layer multicast and broadcast, typically focusing on metrics such as energy consumption ${ }^{[11]}$, the number of transmissions or the overhead in route discovery and management ${ }^{[12]}$. Li et al. ${ }^{[13]}$ considered a residual power routing that aims to maximize the remaining battery energy at each node, while keeping the total energy consumption for the routing is bounded. Liang and Yang ${ }^{[14]}$ recently provided an improved solution for this problem. Thus, the network lifetime is defined as the time of the first node failure ${ }^{[15]}$. Several studies of prolonging a network lifetime have been conducted by researchers. For example, Chang and Tassiulas ${ }^{[16]}$ proposed to maximize the network lifetime through avoiding to use low power nodes and choosing a shortest path in terms of the energy consumption if the packet rate in the network is given in advance.

Apart from minimum Steiner tree approaches ${ }^{[17]}$ and a few local search approaches ${ }^{[18]}$ the MEM problem in the case of omnidirectional antennas has mostly been tackled with heuristics for the Minimum Energy Broadcast (MEB) problem with a subsequent pruning procedure to eliminate unnecessary transmissions. Kang and Poorvendran ${ }^{[19]}$ considered maximizing network lifetime broadcast tree problem in both symmetric and asymmetric networks with the objective to keep the broadcast tree alive as long as possible until the power energy in a node expires. However, Kang and Poovendran ${ }^{[19]}$ focused only on a single broadcast flow and does not address the problem of how individual flows should be routed to maximize the total admissible volume of broadcast/multicast traffic in the presence of inter-flow and intra-flow interference.

Finally, in Toh $^{[20]}$ proposed a conditional max-min battery capacity routing schema which adopts different strategies for different stages of battery energy consumption.

\section{MATERIALS AND METHODS}

\section{The proposed method:}

Formulation problem: The Minimum Energy Multicast (MEM) problem is an NPhard optimization problem both in case of omni-directional and directional antennas. A wireless ad-hoc network with $\mathrm{N}$ nodes is considered in this study, in which every node is equipped with omnidirectional antennas and randomly distributed over a specified region. One particular node is chosen to be the source node which has to broadcast a message to all other nodes in the network. It is well known that path loss accounts for the signal attenuation due to the physical distance between transmitter and receiver in a wireless scenario.

A graph representation for the multicast tree in an ad-hoc network is proposed in $^{[8]}$. Given a connected weighted undirected graph $\mathrm{G}=(\mathrm{V}, \mathrm{E}), \mathrm{V}=\left\{\mathrm{v}_{1}, \mathrm{v}_{2}, \ldots\right.$, $\left.\mathrm{v}_{\mathrm{n}}\right\}$ is the vertex set of $\mathrm{G}$ and the $\mathrm{E}=\left\{\mathrm{e}_{1}, \mathrm{e}_{2}, \ldots, \mathrm{e}_{\mathrm{m}}\right\}$ is a finite set of edges represent the connections between these vertices of G. Let The same concept is applied to the MPM problem here in order to model the broadcast tree in the ad-hoc network. We represent an ad-hoc network by a node-weighted directed graph $\mathrm{G}=(\mathrm{N}, \mathrm{E}$, p), where the vertices in $N=\{1,2, \ldots, N\}$ represent the individual mobile node, $\mathrm{E}$ characterizes the set of transmission links in the network (Fig. 1).

The subgraph of $\mathrm{G}$ can be expressed using a vector $\mathrm{x}=\left\{\mathrm{x}_{1}, \mathrm{x}_{2}, \ldots, \mathrm{x}_{\mathrm{m}}\right\}$ where each element $\mathrm{xi}$ can be defined as follows:

$x i=\left\{\begin{array}{lr}1, \text { if edge e is selected in } & \text { the subgraph } \\ 0, & \text { Otherwise }\end{array}\right.$

The element $\mathrm{qu}$ in $\mathrm{q}$, which denotes the transmission power required of by node $\mathrm{u}$ for its transmission in the multicast tree is given by:

$\mathrm{q}_{\mathrm{u}}=\left\{\begin{array}{lr}\max \left(\mathrm{x}_{\mathrm{uv}}, \mathrm{P}_{\mathrm{uv}}\right) \text { if edge }(\mathrm{u}, \mathrm{v}) \in \mathrm{E} \\ \mathrm{o} & \text { Otherwise }\end{array}\right.$

The corresponding consumption power for a broadcast tree is calculated as:

$$
\mathrm{P}_{\mathrm{T}}=\sum_{\mathrm{u} \in \mathrm{N}} \mathrm{q}_{\mathrm{u}}
$$

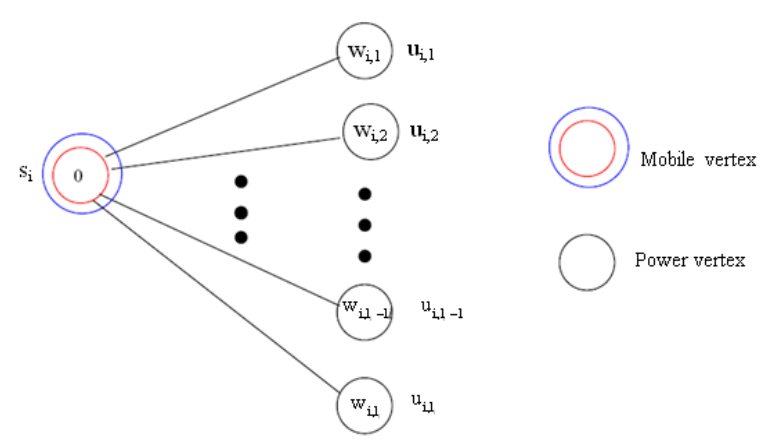

Fig. 1: The widget $G_{i}=\left(V_{i}, E_{i}\right)$ for node $v_{i}$ 
The objective of the MPM is to extract from the original graph $\mathrm{G}$, such that minimum power consumption $\mathrm{P}_{\mathrm{T}^{*}}$. Therefore, the MPB problem can be stated as:

$$
\mathrm{P}_{\mathrm{T}}=\sum_{\mathrm{u} \in \mathrm{N}} \mathrm{q}_{\mathrm{u}}=\sum_{\mathrm{u} \in \mathrm{N}} \max _{\mathrm{v}}\left(\mathrm{x}_{\mathrm{uv}} \mathrm{P}_{\mathrm{uv}}\right)
$$

HPSO for MPM problem: PSO and GA has been successfully used to perform a wide range of optimization problems ${ }^{[10]}$. Each particle in the swarm represents a multicast tree. The population of permutation-based

chromosomes

$\left\{c_{p}=\left[c_{p}^{1}, \ldots . x_{p}^{N}\right], p=1, \ldots, P\right\}$ is initialized by employing a

hybrid of random and deterministic approaches, where $\mathrm{P}$ is known as the population size. In order to produce the deterministic solution, the MPM algorithm is run first and the corresponding node subsequently added to the broadcast tree is recorded into a node sequence, which serves as the deterministic population for the proposed HPSO approach.

Based on the node sequence, the construction of the broadcast tree can be carried out using HPSO algorithm as follows:

Input: Source node $s$ and chromosome $c_{p}=\left[c_{p}^{1}, \ldots . x_{p}^{N}\right]$.

Output: broadcast tree $\mathrm{T}$ and the corresponding consumption power PT. For clarity, the flow of these operations is illustrated in Fig. 2:

- Initialize PT $=0$, mark the source node $\mathrm{s}$ in the permutation $\mathrm{cp}$ to avoid the subsequent selections

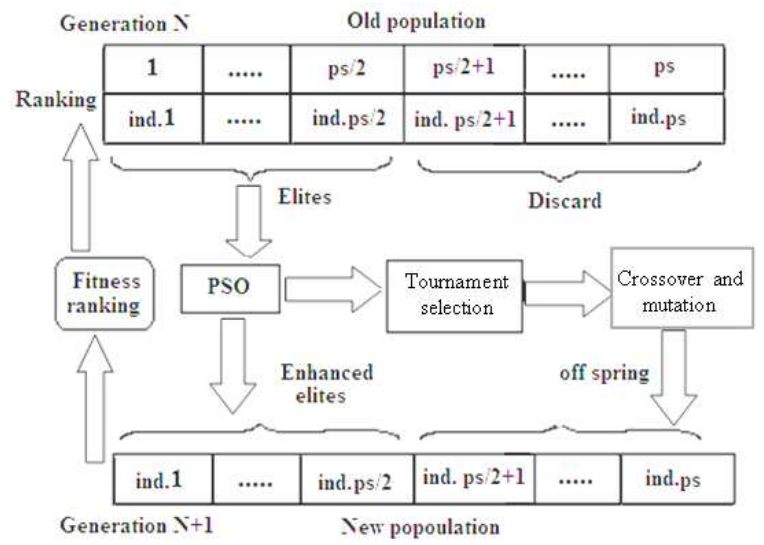

Fig. 2: $\mathrm{HPSO}^{[10]}$
- Obtain the first unmarked node $\mathrm{z}$ in permutation $\mathrm{cp}$, construct a link between source nodes $\mathrm{s}$ and $\mathrm{z}$ and assign the required power psz to the source node

- Check all the remaining unmarked nodes in the permutation to see if any other node is already covered by the above transmission between nodes $s$ and $\mathrm{z}$, mark those nodes to avoid the subsequent selections

- Go through the unmarked node $z^{\prime}$ in permutation sequentially. Employ the rules of the MPM algorithm to add node $\mathrm{z}^{\prime}$ to the existing tree and assign a power $\mathrm{p}_{\mathrm{s}^{\prime} \mathrm{z}^{\prime}}$ to the corresponding source node (assuming it is $\mathrm{s}^{\prime}$ )

- Check all the remaining unmarked nodes in the permutation to see if any other node is covered by the above transmission between nodes $\mathrm{s}^{\prime}$ and $\mathrm{z}^{\prime}$, mark those nodes to avoid subsequent selections

- If not end of permutation, go to 5

- Calculate PT by Eq. 4 and form a multicast tree T in which all constructed links correspond to the edges in $\mathrm{T}$

\section{RESULTS}

In our network model there are 20 nodes including one source node and four sink nodes. And the maximum generation is 80 , the swarm scale is 40 . This part we make a comparison of the simulation results among HPSO, GA. Figure 3 shows the trend of the optimum multicast tree power with maximum generation 80. Figure 4 shows the success rate of HPSO, PSO and GA.

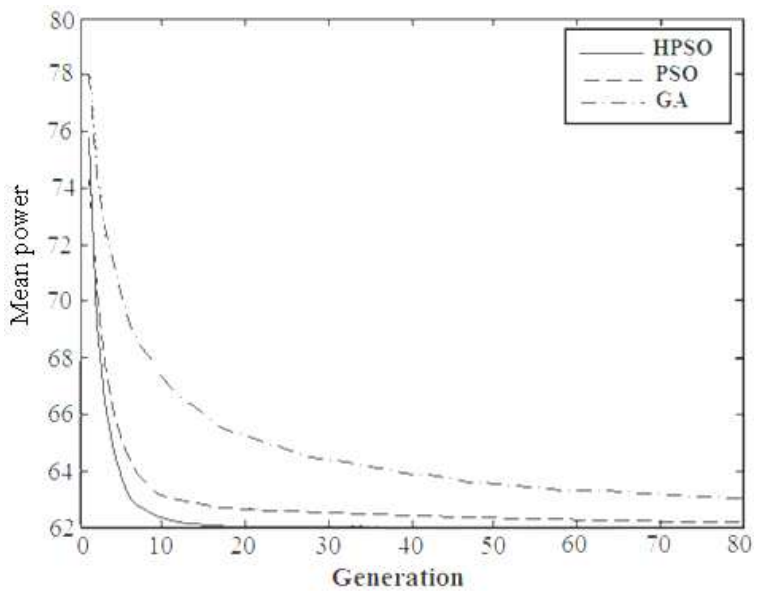

Fig. 3: Multicast tree power with swarm scale 80 


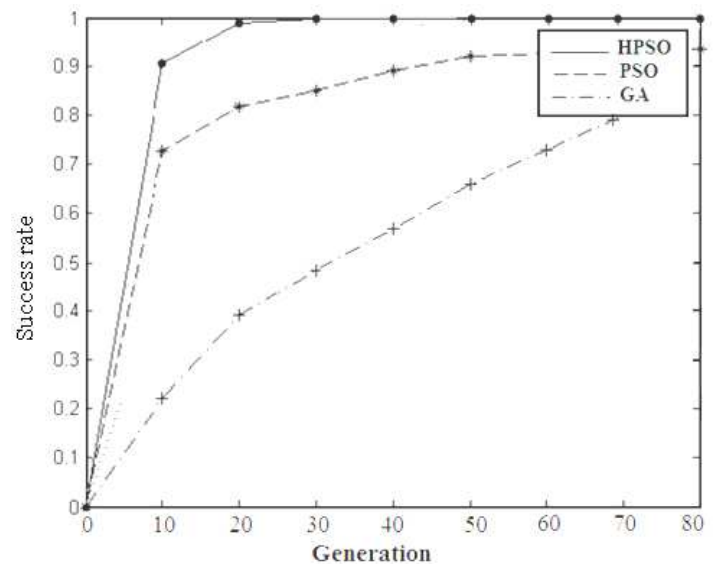

Fig. 4: The success rate

Table 1: Comparison of running time (sec)

\begin{tabular}{llll}
\hline Node & GA & PSO & HPSO \\
\hline 20 & 0.48 & 0.27 & 0.21 \\
40 & 0.63 & 0.45 & 0.38 \\
80 & 1.59 & 1.42 & 1.36 \\
120 & 3.16 & 2.68 & 1.97 \\
160 & 7.72 & 5.37 & 4.18 \\
\hline
\end{tabular}

\section{DISCUSSION}

In Fig. 3, comparing with GA and PSO, the curve of HPSO shows excellent convergence ability in the early stage of iteration and after the 20th generation the algorithm's optimum position is almost found, from 30th-40th generation the particles totally converge to the optimum solution. Comparing with PSO, because of the introducing of probability based updating mechanism, the updating process become more flexible and effective and the searching efficient is improved too. On the other hand, the introducing of the anti-congestion tactics further avoids the happening of immature convergence. It is prove that when the terminal evolution generation is certain, HPSO can find better multicast tree.

In Fig. 4, It show that in the 10th generation the success rate of HPSO exceeds the success rate of GA by $40 \%$ and after 30 th generation, the rate of successful routing is nearly $100 \%$.

The power of each link is uniformly distributed in range $[0,160]$. In Table 1 , we have compared the running times between Hybrid PSO algorithm, PSO and Genetic Algorithm (GA) and for different combinations of node and edge.

\section{CONCLUSION}

Multicast routing problem arises in many multimedia communication applications, computing the band-width-delay constrained least-cost multicast routing tree is a NP-complete problem. In this study introduce multicast power minimization algorithm based on hybrid PSO for multicast algorithm. The experimental results show that this algorithm has performance. The simulation results show that the HPSO combines the advantage of fast convergence, the better global optimization performance and efficiency.

\section{REFERENCES}

1. Xie, J. and X. Wang, 2008. A survey of mobility management in hybrid wireless mesh networks. IEEE Network, 22: 34-40. DOI: 10.1109/MNET.2008.4694172

2. Cagalj, M., J.P. Hubaux and C. Enz, 2002. Minimum-energy broadcast in all wireless networks: NP-completeness and distribution issues. Proceedings of the 8th Annual International Conference on Mobile Computing and Networking, Sept. 23-28, ACM Press, Atlanta, Georgia, USA., $\quad$ pp: 172-182. http://portal.acm.org/citation.cfm?id=570645.570667

3. Wieselthier, J.E., G.D. Nguyen and A. Ephremides, 2000. On the construction of energy-efficient broadcast and multicast trees in wireless networks. Proceedings of the 19th IEEE Annual Joint Conference Computer and Communications Societies, Mar. 26-30, IEEE Xplore Press, Tel Aviv, Israel, pp: 585-594. DOI: 10.1109/INFCOM.2000.832232

4. Draves, R., J. Padhye and B. Zill, 2004. Routing in multi-radio, multi-hop wireless mesh networks, Proceedings of the 10th Annual International Conference on Mobile Computing and Networking, Sept. 26-Oct. 30, ACM Press, Philadelphia, PA., USA., pp: 114-128. http://portal.acm.org/citation.cfm?id=1023732

5. Raniwala, A., K. Gopalan and T.C. Chiueh, 2004. Centralized channel assignment and routing algorithms for multi-channel wireless mesh networks. ACM SIGMOBILE Mobile Comput. Commun. Rev., 8: 50-65. http://portal.acm.org/citation.cfm?id=997122.997130

6. Pettie, S. and V. Ramachandran, 2002. An optimal minimum spanning tree algorithm. J. ACM., 49: $16-34$.

http://portal.acm.org/citation.cfm?id=505243

7. Feeney, L.M. and M. Nilson, 2001. Investigating the energy consumption of a wireless network interface in an ad hoc networking environment. Proceedings of the 20th IEEE Annual Joint Conference Computer and Communications Societies, Mar. 22-26, IEEE Xplore Press, Anchorage, AK., pp: 1548-1557. DOI: 10.1109/INFCOM.2001.916651 
8. Marks, R.J., A.K. Das, M. El-Sharkawi, P. Arabshahi and A. Gray, 2002. Minimum power broadcast trees for wireless networks: Optimizing using the viability lemma. Proceedings of the IEEE International Symposium on Circuits and Systems, May 26-29, IEEE Xplore Press, Scottsdale, AZ., pp: 273-276. DOI: 10.1109/ISCAS.2002.1009830

9. Das, A.K., R.J. Marks, M. El-Sharkawi, P. Arabshahi and A. Gray, 2003. r-shrink: A heuristic for improving minimum power broadcast trees in wireless networks. Proceedings of the IEEE Globecom Telecommunication Conference, Dec. 1-5, IEEE Xplore Press, San Francisco, CA., pp: 523-527. DOI: 10.1109/GLOCOM.2003.1258292

10. Li, C., C. Cao, Y. Li and Y. Yu, 2007. Hybrid of Genetic algorithm and particle swarm optimization for multicast QoS routing. Proceedings of the IEEE International Conference on Control and Automation, May 30-June 1, IEEE Xplore Press, Guangzhou, pp: 2355-2359. DOI: 10.1109/ICCA.2007.4376782

11. Guo, S. and O. Yang, 2004. A dynamic multicast tree reconstruction algorithm for minimum-energy multicasting in wireless ad hoc networks. Proceedings of the 23rd International Performance Computing and Communications Conference, (PCCC'04) IEEE Xplore Press, USA., pp: 637-642. DOI: 10.1109/PCCC.2004.1395119

12. Qadir, J., C.T. Chou and A. Misra, 2006, Exploiting rate diversity for multicasting in multiradio wireless mesh networks. Proceeding of the 31st IEEE Conference on Local Computer Networks, Nov. 14-16, IEEE Xplore Press, Tampa, FL., pp: 287-294. DOI: 10.1109/LCN.2006.322133

13. Hui, X., M. Jeon, S. Lei, N. Yu, J. Cho and S. Lee, 2006. Impact of practical models on power aware broadcast protocols for wireless ad hoc and sensor networks. Proceeding of the 4th IEEE Workshop on Software Technologies for Future Embedded and Ubiquitous Systems and 2nd International Workshop on Collaborative Computing, Integration, and Assurance, Apr. 27-28, IEEE Xplore Press, Gyeongju, pp: 1-6. DOI: 10.1109/SEUS-WCCIA.2006.26
14. Liang, W. and Y. Yang, 2004. Maximizing battery life routing in wireless ad hoc networks. Proceeding of the 37th Hawaii International Conference on System Sciences, Jan. 5-6, IEEE Computer Society, USA., pp: 1-8. DOI: 10.1109/HICSS.2004.1265697

15. Zhang, M., C. Gong and Y. Feng, 2008. An energy-efficient algorithm for maximum lifetime and minimum routing in wireless sensor networks. Proceeding of the International Symposium on Computational Intelligence and Design, Oct. 17-18, IEEE Xplore Press, Wuhan, pp: 507-510. DOI: 10.1109/ISCID.2008.113

16. Chang, J.H. and L. Tassiulas, 2000. Energy conserving routing in wireless ad hoc networks. Proceedings of the 19th IEEE Annual Joint Conference Computer and Communications Societies, Mar. 26-30, IEEE Xplore Press, Tel Aviv, Israel, pp: 22-31. DOI: 10.1109/INFCOM.2000.832170

17. Cartigny, J., D. Simplot and I. Stojmenovic, 2003. Localized minimum-energy broadcasting in ad hoc networks. Proceeding of the 22nd Annual Joint Conference of the IEEE Computer and Communications, Mar. 30-Apr. 3, IEEE Xplore Press, USA., pp: 2210-2217. http://ieeexplore.ieee.org/xpl/freeabs_all.jsp?arnum ber $=1209241$

18. Marks, R.J., A.K. Das, M. El-Sharkawi, P. Arabshahi and A. Gray, 2002. Maximizing lifetime in an energy constrained wireless sensor array using team optimization of cooperating systems. Proceeding of the World Congress Conference on Computational Intelligence, (CI'02), New York, pp: 669-674.

19. Toh, C.K., 2001. Maximum battery life routing to support ubiquitous mobile computing in wireless as hoc networks. IEEE Commun. Mag., 39: 138-147. DOI: $10.1109 / 35.925682$ 